\title{
Competitive Design of Power Allocation Strategies for Energy Harvesting Wireless Communication Systems
}

\author{
Jesus Gomez-Vilardebo \\ Centre Tecnològic de Telecomunicacions de Catalunya (CTTC) \\ Castelldefels, Spain \\ jesus.gomez@cttc.es
}

\begin{abstract}
This paper considers the design of online power allocation strategies for slotted energy harvesting point-to-point communications systems. The objective is to minimize the competitive rate gap that is defined as the maximum gap between the optimal rates that can be achieved by the offline and online policies over all possible energy arrival profiles. A lower-bound on the competitive rate gap is derived and a new online policy is presented. The new online policy is shown to perform very close to the competitive rate gap lower-bound for any number of slots and to outperform previously proposed online policies, such us the myopic policy.
\end{abstract}

\section{INTRODUCTION}

Energy harvesting $(\mathrm{EH})$ technology is considered as a major component of future wireless networks. Harvesting energy from the environment extends the lifetime of wireless devices, and provides them untethered mobility, as batteries can be charged without connecting to the power grid infrastructure. However, despite such advantages, designing EH communication systems bring its own challenges. For many energy sources, such as solar, vibration or electromagnetic, the characteristics of the $\mathrm{EH}$ profile change over time. The time-varying nature of the available energy motivates the need for designing transmission polices that take into account the stochastic nature of the energy arrival process, while optimizing a desired performance criteria.

We model the EH process as a slotted packet arrival process, in which the energy arrives in packets at each time slot, and we study the problem of maximizing the achievable average throughput over a fixed number of time slots. We assume that the energy harvested during the course of the communication is used only in the power amplifier of the transmitter.

Previous work addressing the design of transmission polices for $\mathrm{EH}$ devices are typically classified based on the assumptions made on the transmitter's knowledge about the $\mathrm{EH}$ process [1]. In the offline optimization framework the transmitter is assumed to have access to all the future energy packet arrival instants and packet sizes. The optimal offline transmission policy maximizing the throughput for an $\mathrm{EH}$

This work was partially supported by grant PCIN-2013-027 (E-CROPS), in the frame of the ERA-NET CHISTERA, and the European project FP7ICT-2011-8 NEWCOM\# 318306. point-to-point additive white Gaussian noise (AWGN) channel was first studied in [2] and, later extended to battery capacity constraints and imperfections in [3] and [4], and also to several multi-terminal communications channels, [5]-[9]. Offline designs serves as theoretical upper-bounds and have also been proven useful in inspiring online policies, such us the myopic policy [10]. However, practical interest in offline polices is limited to scenarios for which the EH process is more or less deterministic, or is random, but can be accurately predicted. For example, solar based systems and shoe-mounted piezoelectric devices.

The online optimization framework, instead, assumes that the future energy arrivals are unknown. If the transmitter has statistical knowledge of the underlying EH process then, the optimization problem is modeled as a Markov decision process, and the optimal policy can be determined through dynamic programming [1], [11]. Most of the work in the literature on the online optimization show performance results that are very close to those achieved by optimal offline policies [12], [13]. However, it is not yet clear how much of these results can be attributed to the particular online policy chosen, or the stochastic model considered for the EH process.

In this work, we adopt a competitive analysis framework, for which the statistics of the EH process are not relevant. Our main objective is to characterize the gap between the optimal offline policy and online policy. Identifying this gap independent of the EH statistics will determines the value of the knowledge about the EH process. If the gap between the optimal offline and online policies can be significantly large, more effort should be put into characterizing and learning the behaviour of the underlying EH processes [14]. Moreover, the value of the gap will also let us know the value of the offline results as a performance benchmark, a claim commonly used in the literature.

The most related paper to our work is [15], in which the authors introduce a competitive analysis for an $\mathrm{EH}$ communication systems, and define the competitive rate ratio as the maximum ratio between the optimal offline rate and online rate. They consider point-to-point communications over a slotted transmission interval and consider, both, arbitrary energy arrivals and time-varying channel coefficients which are known only causally at the transmitter. For this scenario, 
authors show that the optimal competitive ratio over $N$ slots is $N$. Here, we study the competitive rate gap rather than the competitive ratio for a static channel setting. In our previous work [16], we have shown that the optimal competitive rate gap for $N$ time slots is upper-bounded by $\log _{2}(N)$ and show that the myopic policy always obtain a lower rate gap. Here, we provide a lower-bound on the competitive rate gap for any number of slots and present a new online policy which performs very close to the lower-bound derived.

The remainder of the paper is organized as follows. The system model is described in Section II. The competitive analysis framework is developed in Section III. The competitive rate gap lower-bound is derived in Section IV, and the new online policy is presented in and evaluated numerically in V. Finally, concluding remarks are presented in Section VI.

\section{SySTEM MOdEL}

Consider the wireless transmission from a source to a destination over a zero mean unit variance circularly symmetric additive white Gaussian noise (AWGN) channel. The communication has a fixed time duration of $T$ time units, which is divided into $N$ slots of equal duration $\frac{T}{N}$. We consider the Shannon capacity function to relate the achieved instantaneous rate to the power; that is, if the transmission power at time $t$ is $p(t)$ then the instantaneous rate is given by $r(p(t))=\log _{2}(1+p(t))$, and the total number of bits transmitted over the period of time $T$ is given by $\int_{0}^{T} r(p(t)) d(t)$.

The source terminal harvests energy from the environment over time. The energy harvested during time slot $n-1$ is first stored into the battery, and is only available at the beginning of slot $n$, and is denoted by $E_{n} \in\left\{0, \mathbb{R}^{+}\right\}, n=1,2, \ldots, N$.

Denote by $U_{n}$ the energy allocated for transmission during time slot $n$. It is well known that, due to the strict concavity of the capacity function, the rate in each slot is maximized by equally distributing the energy $U_{n}$ over the whole slot duration $\frac{T}{N}$. Then, the total number of bits transmitted over slot $n$ is found as follows

$$
D_{n}\left(U_{n}\right)=\frac{T}{N} \log _{2}\left(1+N \frac{U_{n}}{T}\right) .
$$

After $N$ time slots, the rate achieved is $R=$ $\frac{1}{T} \sum_{n=1}^{N} D_{n}\left(U_{n}\right)$. Due to the energy causality constraint, the total energy used by the end of slot $n$ cannot be more than the energy harvested by the beginning of time slot $n$, $n=1,2, \ldots, N$, that is, $U_{m}$ values have to satisfy:

$$
\sum_{m=1}^{n} U_{m} \leq \sum_{m=1}^{n} E_{m}, \forall n \leq N .
$$

Hereafter, without loss of generatively, we consider $T=1$.

\section{COMPETITIVE ANALYSis}

Our goal is to study the rate gap between the rate achieved by the optimal offline policy $R_{O}(\mathbf{E})$, which was first presented in [2], and the rate $R_{\mathcal{U}}(\mathbf{E})$ achieved by the online policy maximized over all possible energy profiles. We want to characterize the minimum value of this maximum rate gap, the competitive rate gap $(g)$, defined as

$$
g=\min _{\mathcal{U}} \max _{\mathbf{E} \in\left\{0, \mathbb{R}^{+}\right\}^{N}} R_{O}(\mathbf{E})-R_{\mathcal{U}}(\mathbf{E}) .
$$

For the minimization, we consider online policies $\mathcal{U}$ that make their decisions based only on the past energy arrivals, and make no assumption about the statistics of the EH process, namely

$$
\mathcal{U}\left(\left\langle E_{1}, \ldots, E_{N}\right\rangle\right)=\left\langle U_{1}, \ldots, U_{N}\right\rangle,
$$

where the energies spent at time slots $n=1, \ldots, N$ are defined by the functions

$$
U_{n}\left(\left\langle E_{1}, \ldots, E_{n}\right\rangle\right):\left\{0, \mathbb{R}^{+}\right\}^{n} \rightarrow\left[0, B_{n}\right],
$$

where $\mathbf{E}_{n}=\left\langle E_{1}, \ldots, E_{n}\right\rangle$ and $B_{n}$ denotes the amount of energy in the battery at the beginning of time slot $n$. Notice that $U_{n}$ is a fraction of $B_{n}$, which we can write for convenience as

$$
U_{n}\left(\mathbf{E}_{n}\right)=\alpha_{n}\left(\mathbf{E}_{n}\right) B_{n}
$$

where $0<\alpha_{n}\left(E_{n}\right) \leq 1$ and the battery state at time slot $n$, can be computed recursively as

$$
B_{n}=\left(1-\alpha_{n-1}\right) B_{n-1}+E_{n} .
$$

In general, solving (1) directly can be quite difficult, we have instead derived upper- and lower-bounds on $g$. Upperbounds can be obtained by fixing a particular online policy $\mathcal{U}^{*}$ and solving instead

$$
g_{U}=\max _{E^{N} \in \mathcal{E}} R_{O}-R_{\mathcal{U}^{*}}
$$

By using this approach, we have shown in [16], that the competitive rate gap is upper-bounded as

$$
g \leq \log _{2} N
$$

and that, the myopic policy

$$
U_{n}=\sum_{l=1}^{n} \frac{E_{l}}{N-l+1}, n=1, \ldots, N
$$

which uses the offline optimization solution on the current available energy as if there will be no further energy arrivals is able to obtain a lower competitive rate gap.

Here, we derive a lower-bound on the competitive rate gap and present an online policy, which is shown to obtain a competitive rate gap very close to the lower-bound, outperforming the rate gap obtained with the myopic policy.

\section{Competitive Rate Gap Lower Bound}

To find a lower-bound on the competitive rate gap (1), we maximize the rate gap only over a subset of all the possible EH input sequences $\mathcal{S} \subset\left\{0, \mathbb{R}^{+}\right\}^{N}$, namely

$$
g \geq \min _{\mathcal{U}} \max _{\mathbf{E} \in S} R_{O}(\mathbf{E})-R_{\mathcal{U}}(\mathbf{E}) .
$$

We consider a particular subset of $2^{N-1}$ EH input sequences of length $N$. To refer to each of these sequences, we use a length- $N$ vector of binary entries $\mathbf{x}=\langle\mathbf{x}[1], \ldots, \mathbf{x}[N]\rangle$, with 
$\mathbf{x}[1]=1$ and $\mathbf{x}[n] \in\{0,1\}$ for $n=2, \ldots, N$, and define the EH sequences associated to $\mathbf{x}$, as

$$
\mathbf{E}_{\mathbf{x}}[n]= \begin{cases}\lim _{E_{1} \rightarrow \infty}\left(E_{1}\right)^{n}, & \text { if } \mathbf{x}[n]=1 \\ 0, & \text { if } \mathbf{x}[n]=0\end{cases}
$$

For each of these EH input sequences, the online rate is denoted as $R_{\mathcal{U} \mid \mathbf{x}}=R_{\mathcal{U}}\left(\mathbf{E}_{\mathbf{x}}\right)$, and given by

$$
R_{\mathcal{U} \mid \mathbf{x}}=\frac{1}{N} \sum_{n=1}^{N} \log _{2}\left(1+N U_{n \mid \mathbf{x}_{n}}\right)
$$

where $U_{n \mid \mathbf{x}_{n}}=U_{n}\left(\mathbf{E}_{\mathbf{x}_{n}}\right)$ denotes the energy allocated at time slot $n$, which can be found as

$$
U_{n \mid \mathbf{x}_{n}}=\alpha_{n}\left(\mathbf{E}_{\mathbf{x}_{n}}\right) B_{n}
$$

The optimal offline rate obtained with $\mathbf{E}_{\mathbf{x}}$ is denotes as $R_{O \mid \mathbf{x}}$. For the EH input sequences in (6), it can be shown that, the optimal offline transmission policy [2] consists of spending the energy received at time slot $n$, when $\mathbf{x}[n]=1$, equally among the next consecutive slots $m>n$ satisfying $\mathbf{x}[m]=0$.

The rate gap associated to $\mathbf{E}_{\mathbf{x}}$ is denoted as $G_{\mathbf{x}}=G_{\mathbf{x}}\left(\mathbf{E}_{\mathbf{x}}\right)$, and given by

$$
G_{\mathbf{x}}=R_{O \mid \mathbf{x}}-R_{\mathcal{U} \mid \mathbf{x}}
$$

Finally, we can compute the competitive rate gap lower-bound, by solving

$$
\begin{aligned}
g_{L}= & \min _{\alpha_{n \mid \mathbf{x}_{n}} \max _{\mathbf{x}} G_{\mathbf{x}}} \\
& 0 \leq \alpha_{n \mid \mathbf{x}_{n}} \leq 1, \forall \mathbf{x}_{n} \text { and } n=1, \ldots, N .
\end{aligned}
$$

For the sake of clarity, we solve (7) first for $N=3$, and then extend the result to any number of slots $N$.

For $N=3$, the $\mathrm{EH}$ input sequences defined in (6) are given by: $\mathbf{E}_{1,0,0}=\lim _{E_{1} \rightarrow \infty}\left\langle E_{1}, 0,0\right\rangle, \mathbf{E}_{1,0,1}=$ $\lim _{E_{1} \rightarrow \infty}\left\langle E_{1}, 0, E_{1}^{3}\right\rangle, \quad \mathbf{E}_{1,1,0}=\lim _{E_{1} \rightarrow \infty}\left\langle E_{1}, E_{1}^{2}, 0\right\rangle$, and $\mathbf{E}_{1,1,1}=\lim _{E_{1} \rightarrow \infty}\left\langle E_{1}, E_{1}^{2}, E_{1}^{3}\right\rangle$. The online rates associated to each of these EH input sequences are given by

$$
\begin{aligned}
& R_{\mathcal{U} \mid 1,0,0}=\lim _{E_{1} \rightarrow \infty} \frac{1}{3} \log _{2}\left(3^{3} \alpha_{1 \mid 1} \alpha_{2 \mid 1,0} \alpha_{3 \mid 1,0,0}\right. \\
& \left.\quad\left(1-\alpha_{1 \mid 1}\right)^{2}\left(1-\alpha_{2 \mid 1,0}\right) E_{1}^{3}\right), \\
& R_{\mathcal{U} \mid 1,0,1}=\lim _{E_{1} \rightarrow \infty} \frac{1}{3} \log _{2}\left(3^{3} \alpha_{1 \mid 1} \alpha_{2 \mid 1,0} \alpha_{3 \mid 1,0,1}\left(1-\alpha_{1 \mid 1}\right) E_{1}^{5}\right), \\
& R_{\mathcal{U} \mid 1,1,0}=\lim _{E_{1} \rightarrow \infty} \frac{1}{3} \log _{2}\left(3^{3} \alpha_{1 \mid 1} \alpha_{2 \mid 1,1} \alpha_{3 \mid 1,1,0}\left(1-\alpha_{2 \mid 1,1}\right) E_{1}^{5}\right), \\
& R_{\mathcal{U} \mid 1,1,1}=\lim _{E_{1} \rightarrow \infty} \frac{1}{3} \log _{2}\left(3^{3} \alpha_{1 \mid 1} \alpha_{2 \mid 1,1} \alpha_{3 \mid 1,1,1} E_{1}^{6}\right),
\end{aligned}
$$

the offline rates are given by

$$
\begin{aligned}
& R_{O \mid 0,0}=\lim _{E_{1} \rightarrow \infty} \frac{1}{3} \log _{2}\left(3^{3} E_{1}^{3}\right), \\
& R_{O \mid 0,1}=\lim _{E_{1} \rightarrow \infty} \frac{1}{3} \log _{2}\left(\frac{3^{3}}{2^{2}} E_{1}^{5}\right), \\
& R_{O \mid 1,0}=\lim _{E_{1} \rightarrow \infty} \frac{1}{3} \log _{2}\left(\frac{3^{3}}{2^{2}} E_{1}^{5}\right), \\
& R_{O \mid 1,1}=\lim _{E_{1} \rightarrow \infty} \frac{1}{3} \log _{2}\left(3^{3} E_{1}^{6}\right),
\end{aligned}
$$

and the rate gaps are

$$
\begin{aligned}
G_{1,0,0} & =-\frac{1}{3} \log _{2}\left(3^{3} \alpha_{1 \mid 1} \alpha_{2 \mid 1,0} \alpha_{3 \mid 1,0,0}\left(1-\alpha_{1 \mid 1}\right)^{2}\left(1-\alpha_{2 \mid 1,0}\right)\right), \\
G_{1,0,1} & =-\frac{1}{3} \log _{2}\left(2^{2} \alpha_{1 \mid 1} \alpha_{2 \mid 1,0} \alpha_{3 \mid 1,0,1}\left(1-\alpha_{1 \mid 1}\right)\right) \\
G_{1,1,0} & =-\frac{1}{3} \log _{2}\left(2^{2} \alpha_{1 \mid 1} \alpha_{2 \mid 1,1} \alpha_{3 \mid 1,1,0}\left(1-\alpha_{2 \mid 1,1}\right)\right) \\
G_{1,1,1} & =-\frac{1}{3} \log _{2}\left(\alpha_{1 \mid 1} \alpha_{2 \mid 1,1} \alpha_{3 \mid 1,1,1}\right) .
\end{aligned}
$$

Substituting $G_{\mathbf{x}}$ for all $\mathbf{x}$ into (7), it can be shown that the minimum rate gap is obtained in the equality of all the rate gaps $G_{1,0,0}=G_{1,0,1}=G_{1,1,0}=G_{1,1,1}$. By solving the resultant system of equations, we obtain

$$
\begin{aligned}
\alpha_{3 \mid 1,1,1} & =\alpha_{3 \mid 1,1,0}=\alpha_{3 \mid 1,0,1}=\alpha_{3 \mid 1,0,0}=1 \\
\alpha_{2 \mid 1,1} & =1-\Gamma_{2} \alpha_{3 \mid 1,1,1} \\
\alpha_{2 \mid 1,0} & =1-\frac{\Gamma_{3} \alpha_{3 \mid 1,0,1}}{1-\alpha_{1 \mid 1}} \\
\alpha_{1 \mid 1} & =1-\Gamma_{2} \alpha_{2 \mid 1,1}-\Gamma_{3} \alpha_{3 \mid 1,1,1}
\end{aligned}
$$

where $\Gamma_{l}=\frac{(l-1)^{l-1}}{l^{l}}$. Finally, given that the rate gaps are equal for all $\mathbf{x}$, we chose $\mathbf{x}=\langle 1,1,1\rangle$ obtaining

$$
\begin{aligned}
g_{L} & =-\frac{1}{3} \log _{2}\left(\alpha_{1 \mid 1} \alpha_{2 \mid 1,1} \alpha_{3 \mid 1,1,1}\right), \\
& =-\frac{1}{3} \log _{2}\left(1-\Gamma_{2}\right)-\frac{1}{3} \log _{2}\left(1-\Gamma_{2}\left(1-\Gamma_{2}\right)-\Gamma_{3}\right), \\
& =0.33501 \text { bits/frame. }
\end{aligned}
$$

For $N$ time slots, it can be shown that, the gap associated to the $\mathrm{EH}$ input sequence $\mathbf{E}_{\mathbf{x}}$ is given by

$$
\begin{gathered}
G_{\mathbf{x}}=-\frac{1}{N} \log _{2}\left(\prod_{n=1}^{N} \alpha_{n \mid \mathbf{x}_{n}} \prod_{i=1}^{\left|z_{\mathbf{x}}\right|}\left(\mathbf{z}_{\mathbf{x}}(i)+1\right)^{\mathbf{z}_{\mathbf{x}}(i)+1}\right. \\
\left.\prod_{j=1}^{z_{\mathbf{x}}(i)}\left(1-\alpha_{\mathbf{o}_{x}(j) \mid \mathbf{x}_{j}}\right)^{\mathbf{z}_{\mathbf{x}}(i)+1-j}\right)
\end{gathered}
$$

where $\mathbf{o}_{\mathbf{x}}$ is a vector containing the position of each one in $\mathbf{x}$ and $\mathbf{z}_{\mathbf{x}}$ is a vector with the number of consecutive zeros after each one. As an example, for $\mathbf{x}=\langle 1,0,0,1,0,1\rangle, \mathbf{o}_{x}=[1,4,6]$ and $\mathbf{z}_{\mathbf{x}}=[2,1]$.

Solving the system of equations defined by $G_{\mathbf{x}}=G_{\mathbf{y}}$ for all $\mathbf{y} \neq \mathbf{x}$, we have found the following solution to all $\alpha_{n \mid \mathbf{x}_{n}}$ and the competitive rate gap lower-bound: Let us denote the last position of a one in $\mathbf{x}_{n}$ as $m\left(\mathbf{x}_{n}\right)$. For any two distinct 
binary input vectors $\mathbf{x}_{n}$ and $\mathbf{y}_{n}$, if the last position of a one in $\mathbf{x}_{n}$ and in $\mathbf{y}_{n}$ coincide, $m\left(\mathbf{x}_{n}\right)=m\left(\mathbf{y}_{n}\right)$, then

$$
\alpha_{n \mid \mathbf{x}_{n}}=\alpha_{n \mid \mathbf{y}_{n}} .
$$

Observe that this equality implies that there are $n$ distinct $\alpha_{n}$ instead of the $2^{n-1}$ possible $\alpha_{n \mid \mathbf{x}_{n}}$. We define

$$
\alpha_{n \mid m\left(\mathbf{x}_{n}\right)}^{*}=\alpha_{n \mid \mathbf{x}_{n}}
$$

and compute $\alpha_{n \mid m}^{*}$, for $m \leq n$, and $n=1, \ldots, N$, by first obtaining $\alpha_{n \mid n}^{*}$, iteratively, for $n=N$ to $n=1$ using

$$
\alpha_{n \mid n}^{*}=1-\sum_{l=2}^{N+1-n} \Gamma_{l} \alpha_{n+l-1 \mid n+l-1}^{*} .
$$

For $m<n$, we computed $\alpha_{n \mid m}^{*}$, recursively, for $n=2$ to $n=N$ and for $m=1$ to $m=n-1$, as

$$
\begin{gathered}
\alpha_{n \mid m}^{*}=1-\prod_{l=m}^{n-1}\left(1-\alpha_{l \mid m}^{*}\right)^{-1} \\
\sum_{l=2}^{N+1-n} \Gamma_{l+n-m} \alpha_{n+l-1 \mid n+l-1}^{*} .
\end{gathered}
$$

Finally, by choosing $\mathbf{x}$ to be the vector of all ones, namely $\mathbf{x}=\mathbf{1}_{N-1}$, we obtain

$$
\begin{aligned}
g_{L} & =-\frac{1}{N} \log _{2}\left(\prod_{n=1}^{N} \alpha_{n \mid \mathbf{x}_{n}}\right), \\
& =-\frac{1}{N} \log _{2}\left(\prod_{n=1}^{N} \alpha_{n \mid n}^{*}\right) .
\end{aligned}
$$

\section{Online Policy}

Online policies consider that future energy arrivals are unknown. The online rate maximization problem falls into the category of Markov decision processes and the optimal solution can be identified by dynamic programming. However, the complexity of finding the solution with dynamic programming grows exponentially with the variables of the system, i.e. number of slots, possible energy arrival amounts, etc. Simple online policies have also been proposed in the literature without claiming optimality, such as the greedy policy, which uses all the available energy in the next slot, $U_{n}=E_{n}$, or the myopic policy (4). Here, we present a new simple online policy designed to approach the competitive gap lower-bound in (11).

At each time slot $n$, using only the EH input sequence up to time slot $n$, the online police decides on the fraction $\alpha_{n}\left(\mathbf{E}_{n}\right)$ of the remaining energy in the battery $B_{n}$ to be spent at time slot $n$, namely

$$
U_{n}\left(\mathbf{E}_{n}\right)=\alpha_{n}\left(\mathbf{E}_{n}\right) B_{n} .
$$

At time slot $n, \alpha_{n}\left(\mathbf{E}_{n}\right)$ is chosen as one of the $n$ possible energy fractions $\alpha_{n \mid m} m=1, . ., n$ defined in (9) and (10). Observe that all $\alpha_{n \mid m}, m \leq n$, and $n=1, \ldots, N$ can be precomputed and stored in a look-up table, as done in Table I, for $N=5$.

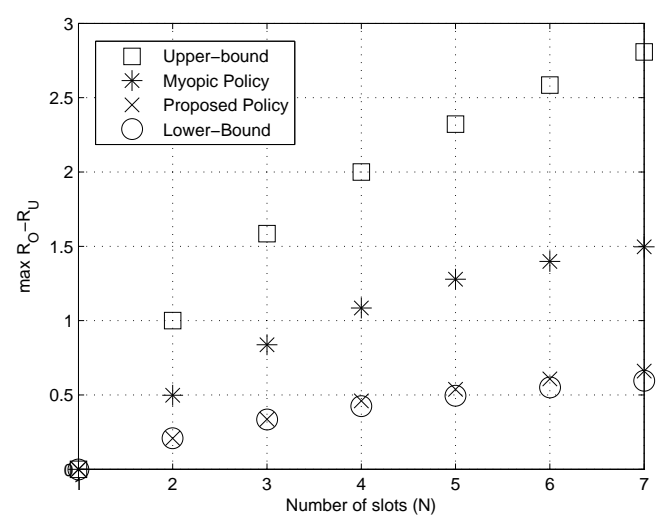

Fig. 1: Competitive rate gap analysis

TABLE I: Look-up table for the online policy with $N=5$

$\begin{array}{cccccc}\mathrm{m} & \alpha_{1, m} & \alpha_{2, m} & \alpha_{3, m} & \alpha_{4, m} & \alpha_{5, m} \\ 1 & 0.5862 & 0.3730 & 0.3794 & 0.4912 & 1 \\ 2 & & 0.6173 & 0.4340 & 0.5130 & 1 \\ 3 & & & 0.6644 & 0.5586 & 1 \\ 4 & & & & 0.7500 & 1 \\ 5 & & & & & 1\end{array}$

At time slot $n=1$, there is only one possible energy fraction, and thus $\alpha_{1}\left(E_{1}\right)=\alpha_{1 \mid 1}$ for all $E_{1}$. At time slot $n>1$, suppose that $\alpha_{n-1}\left(\mathbf{E}_{n-1}\right)=\alpha_{n \mid m}$ then, the policy evaluates the following condition

$$
E_{n} \geq \frac{1}{n-m+1} \sum_{i=m}^{n} E_{i},
$$

and selects

$$
\alpha_{n}\left(\mathbf{E}_{n}\right)= \begin{cases}\alpha_{n \mid n} & \text { if (13) satisfied, } \\ \alpha_{n \mid m} & \text { otherwise. }\end{cases}
$$

We conclude this section by providing a numerical assessment of the competitive rate gap for $N=2, \ldots, 7$ in Fig.1. To that end, we limit the energy harvesting amounts to the discrete set $E_{n} \in\left[0,10,20, \ldots, 10^{5}\right]$, and compute the competitive rate gap obtained with the myopic policy and with the online policy here presented. The lower-bound in (11) and the upper-bound in (3) are also depicted. Observe that, as opposed to the myopic policy, the proposed online strategy is able to achieve the competitive rate gap lower-bound for $N \leq 3$, and is very close to the rate gap lower-bound for any $N$.

\section{CONCLUSIONS}

We studied the competitive rate gap for EH communication systems. For slotted point-to-point communications, we provided a lower-bound on the competitive rate gap, which is defined as the maximum difference between the rate obtained with an offline power policy and an online power policy. Based on the derivation of this lower-bound, we proposed an online strategy, which is shown, via numerical evaluation, to obtain a rate gap very close to the rate gap lower-bound for any number of slots. 


\section{REFERENCES}

[1] D. Gunduz, K. Stamatiou, N. Michelusi, and M. Zorzi, "Designing intelligent energy harvesting communication systems," Communications Magazine, IEEE, vol. 52, no. 1, pp. 210-216, January 2014.

[2] J. Yang and S. Ulukus, "Optimal packet scheduling in an energy harvesting communication system," Communications, IEEE Transactions on, vol. 60, no. 1, pp. 220-230, January 2012.

[3] K. Tutuncuoglu and A. Yener, "Optimum transmission policies for battery limited energy harvesting nodes," Wireless Communications, IEEE Transactions on, vol. 11, no. 3, pp. 1180-1189, March 2012.

[4] B. Devillers and D. Gunduz, "A general framework for the optimization of energy harvesting communication systems with battery imperfections," Communications and Networks, Journal of, vol. 14, no. 2, pp. 130-139, April 2012.

[5] O. Ozel, J. Yang, and S. Ulukus, "Optimal broadcast scheduling for an energy harvesting rechargeable transmitter with a finite capacity battery," Wireless Communications, IEEE Transactions on, vol. 11, no. 6, pp. 2193-2203, June 2012.

[6] J. Yang and S. Ulukus, "Optimal packet scheduling in a multiple access channel with energy harvesting transmitters," Communications and Networks, Journal of, vol. 14, no. 2, pp. 140-150, April 2012.

[7] K. Tutuncuoglu and A. Yener, "Sum-rate optimal power policies for energy harvesting transmitters in an interference channel," Communications and Networks, Journal of, vol. 14, no. 2, pp. 151-161, April 2012.

[8] D. Gunduz and B. Devillers, "Two-hop communication with energy harvesting," in Computational Advances in Multi-Sensor Adaptive Processing (CAMSAP), 2011 4th IEEE International Workshop on, Dec 2011, pp. 201-204.

[9] O. Orhan and E. Erkip, "Throughput maximization for energy harvesting two-hop networks," in Information Theory Proceedings (ISIT), 2013 IEEE International Symposium on, July 2013, pp. 1596-1600.

[10] O. Ozel, K. Tutuncuoglu, J. Yang, S. Ulukus, and A. Yener, "Transmission with energy harvesting nodes in fading wireless channels: Optimal policies," Selected Areas in Communications, IEEE Journal on, vol. 29, no. 8, pp. 1732-1743, September 2011.

[11] C. K. Ho and R. Zhang, "Optimal energy allocation for wireless communications with energy harvesting constraints," Signal Processing, IEEE Transactions on, vol. 60, no. 9, pp. 4808-4818, Sept 2012.

[12] O. Ozel and S. Ulukus, "Achieving awgn capacity under stochastic energy harvesting," Information Theory, IEEE Transactions on, vol. 58, no. 10, pp. 6471-6483, Oct 2012.

[13] M. Kashef and A. Ephremides, "Optimal packet scheduling for energy harvesting sources on time varying wireless channels," Communications and Networks, Journal of, vol. 14, no. 2, pp. 121-129, April 2012.

[14] P. Blasco, D. Gunduz, and M. Dohler, "A learning theoretic approach to energy harvesting communication system optimization," Wireless Communications, IEEE Transactions on, vol. 12, no. 4, pp. 1872-1882, April 2013.

[15] R. Vaze, R. Garg, and N. Pathak, "Dynamic power allocation for maximizing throughput in energy-harvesting communication system," pp. 1-1, 2013

[16] J. Gomez-Vilardebo and D. Gunduz, "Competitive analysis of energy harvesting wireless communication systems," in European Wireless (EW) Conference, May 2014. 